\title{
Restauración psicológica y naturaleza urbana: algunas implicaciones para la salud mental
}

\author{
Joel Martínez-Soto, ${ }^{1}$ María Montero-López Lena, ${ }^{2}$ Ana Córdova y Vázquez ${ }^{3}$
}

Artículo original

\section{SUMMARY}

Topics relating to mental health promotion systematically lack research that documents the importance of physical environment qualities in the development of public health policies. At the present time, several emerging research lines regarding restorative environments (RE) and psychological restoration (PR) may contribute to the conceptual and operational definition of relevant environmental qualities promoting mental health. PR relates to the recovery of people's cognitive resources and psychophysiological responses when exposed to environments with restorative qualities. The present study documents the application of an ecological social model regarding the impact of urban nature on PR in a housing context. We posed the objective to document possible restoration effects of variables related to Urban Nature-UN (for example, views of nature from housing windows, indoor plants and gardens, proximity to outdoor green areas) and Psychological Transaction Processes with the Environment - PTRAPE (activities in nature and perception of environmental restoration of housing) on emotional and cognitive indicators of PR. To achieve this goal, we carried out a study with 120 individual home interviews (mean age of 45.73 years, 63 men and 57 women) in the metropolitan area of Mexico City. The structural equations model showed that UN has both direct and indirect effects on emotional and cognitive dimension of PR. This theoretical, conceptual, and methodological approach provides a conceptual platform to carry out innovative research with relevant empirical implications for mental health promotion.

Key words: Restorative environments, public health, stress, environmental perception.

\section{RESUMEN}

En las temáticas de promoción de salud mental existe una carencia sistemática de investigaciones que documenten la importancia de las cualidades del ambiente físico en el desarrollo de políticas públicas de salud. Actualmente existen varias líneas emergentes de investigación sobre ambientes restauradores (AR) y restauración psicológica (RP) que pueden contribuir a la definición conceptual y operativa de las cualidades del ambiente que pueden ser relevantes para promover la salud mental. La RP se relaciona con la recuperación de los recursos cognitivos y de la capacidad de respuesta psicofisiológica que experimentan las personas ante la exposición a ambientes con cualidades restauradoras. En el presente estudio se documenta la aplicación de un modelo ecológico social del impacto de la naturaleza urbana en la $\mathrm{RP}$ considerando un contexto de vivienda. Se planteó como objetivo documentar los posibles efectos restauradores de variables relacionadas con la presencia de Naturaleza Urbana-NU (e.g. vistas de naturaleza en las ventanas de las viviendas, plantas y jardines interiores, proximidad de áreas verdes exteriores), Procesos Psicológicos de Transacción con el Medio Ambiente - PPTRAMA (actividades con la naturaleza y percepción de restauración ambiental de la vivienda) sobre indicadores emotivos y cognitivos de la RP. Para conseguir tal objetivo se ha llevado a cabo un estudio en el que se entrevistaron en sus domicilios a 120 personas (edad media 45.73 años, 63 hombres y 57 mujeres) de la Zona Metropolitana de la Ciudad de México. Por medio de un modelo de ecuaciones estructurales se comprobó que la NU tiene efectos directos e indirectos en la dimensión emotiva y cognitiva de la RP. Del abordaje teórico, conceptual y metodológico se desprende una plataforma conceptual a partir de la cual es posible desarrollar investigación innovadora con implicaciones empíricas relevantes para la promoción de la salud mental.

Palabras clave: Ambientes restaurativos, salud pública, estrés, percepción ambiental.

\section{INTRODUCCIÓN}

Los problemas sociales y estresores ambientales irrumpen con mayor frecuencia en escenarios altamente urbanizados ${ }^{1}$ e inciden, a su vez, en los trastornos psiquiátricos (e.g. esquizofrenia y depresión). ${ }^{2}$ Se estima que una de cada cuatro personas padece un trastorno mental o neurológico en algún momento de su vida y unos 450 millones de personas sufren

\footnotetext{
Departamento de Psicología, Universidad de Guanajuato.

Facultad de Psicología, Universidad Nacional Autónoma de México.

El Colegio de la Frontera Norte.
}

Correspondencia: Joel Martínez-Soto. Blvd. Puente del Milenio 1001, Fracc. del Predio San Carlos, 37670, León, Gto., México. Teléfono: (477) 267-4900 ext. 3635. Celular: (442) 106-8149. E-mail: masjmx@yahoo.com.mx; jmartinezsoto@ugto.mx

Recibido primera versión: 13 de febrero de 2013. Recibido segunda versión: 26 de febrero de 2014. Aceptado: 5 de marzo de 2014. 
actualmente estos trastornos. ${ }^{3}$ En México, la Encuesta Nacional de Epidemiología Psiquiátrica ${ }^{4}$ reporta que un $9.2 \%$ de la población ha tenido un trastorno depresivo en la vida. Estados emocionales negativos vinculados con el estrés (e.g. ansiedad y hostilidad) han sido relacionados de manera consistente con enfermedades del corazón. ${ }^{5}$ Asimismo, diversas enfermedades crónicas como el cáncer, altos niveles de presión sanguínea y diabetes tipo II tienen una conexión con el estrés. ${ }^{6}$ El estrés, síndrome de la modernización urbana, origina gastos anuales en consultas y tratamientos médicos alrededor del mundo de al menos 100 billones de dólares americanos. $^{*, 7}$ Lo anterior implica una inversión en salud pública potencialmente amenazantes para la economía de las comunidades. ${ }^{8,9}$

La Organización Mundial de la Salud (OMS) suele definir a la salud como un estado de completo bienestar físico, mental y social, y no sólo la ausencia de afecciones o enfermedades. ${ }^{* *}$ La salud mental es un concepto multidimensional que involucra el equilibrio emocional y mental interno, el bienestar subjetivo, la percepción de la propia eficacia o funcionamiento efectivo, la capacidad de encarar adecuadamente el estrés, la autonomía, la autorrealización de las capacidades intelectuales y la capacidad de convivir armoniosamente con los semejantes. ${ }^{10}$ Hoy en día resulta paradójico que esta perspectiva de salud mental positiva tenga un menor eco en los modelos de salud mental, destacándose entre éstos los modelos de enfermedad. ${ }^{11,12}$ Los fundamentos de la salud mental se relacionan con la promoción del bienestar, la prevención de trastornos mentales y el tratamiento y rehabilitación de las personas afectadas por dichos trastornos. ${ }^{13}$ Estas bases suelen tener una orientación hacia los comportamientos de cuidado de salud individual (e.g. hábitos de salud individuales y estilos de vida), ${ }^{14}$ consideran a la salud mental como el resultado de la armonía entre los aspectos biológicos y sociales del individuo ${ }^{15}$ y tienden a enfocarse en menor grado en el ambiente físico como fuente promotora de salud. ${ }^{16}$ Las políticas públicas de salud mental reconocen el rol de la acción combinada de numerosos factores biológicos, psicológicos, sociales y ambientales que determinan que un individuo disfrute de un estado de salud psíquica o que sea afligido por trastornos mentales y del comportamiento. ${ }^{15} \mathrm{Si}$ bien en dichas políticas quedan claros los mecanismos biológicos, psicológicos y sociales a trabajar para promover la salud, ${ }^{17}$ no obstante poco se ha documentado qué factores del ambiente físico ${ }^{18}$ (no sociales) pueden repercutir positivamente (y no sólo negativamente) en la salud mental. En este sentido existen varias líneas emergentes de investigación que, desde la psicología y otras disciplinas afines, pueden contribuir significativamente a la definición conceptual y operativa de

\footnotetext{
* Un billón en los E.U. equivale a mil millones.

* Preámbulo de la Constitución de la Organización Mundial de la Salud, adoptado por la Conferencia Sanitaria Internacional firmada en Nueva York el 22 de julio de 1946 por los representantes de 61 Estados.
}

las cualidades físicas del ambiente que son relevantes para promover la salud mental. ${ }^{19}$

Hoy en día existen suficientes evidencias científicas para promover la existencia de ambientes cuyas cualidades pueden ser catalizadoras de procesos psicológicos vinculados con la salud mental. Diversos estudios han demostrado que el contacto con la naturaleza puede contribuir a reducir el estrés y a promover estados del humor positivos $\mathrm{y}$ un adecuado funcionamiento cognitivo (atencional). ${ }^{20-23}$ Estos efectos, denominados restauradores, han sido documentados ampliamente en la literatura sobre percepción de restauración ambiental ${ }^{24,25}$ y de RP. ${ }^{26,27}$ La Restauración Psicológica (RP en adelante) se relaciona con la recuperación de los recursos cognitivos y de la capacidad de respuesta psicofisiológica que experimentan las personas ante la exposición a ambientes con cualidades restauradoras. ${ }^{28} \mathrm{Al}$ contexto donde ocurre dicha recuperación se le denomina ambiente restaurador y a las dimensiones físicas y psicológicas que contribuyen a que un ambiente sea percibido como restaurador se les denominan cualidades restauradoras. ${ }^{24}$ Estas cualidades pueden variar en función del tipo de ambiente evaluado. Las personas tienden a atribuir más cualidades restauradoras a los ambientes naturales que a los ambientes urbanos sin naturaleza. Entre los escenarios urbanos, aquéllos con presencia de naturaleza urbana (áreas verdes, jardines, parques, vistas de ventanas con contenidos vegetales, etc.) promueven un mayor potencial restaurador al compararse con los que no la poseen. ${ }^{29}$ En la bibliografía sobre los efectos restauradores de la naturaleza urbana se identifican cuatro variables ambientales que son críticas para promover un proceso de RP: a) la visibilidad de contenido vegetal apreciable a través de las ventanas, ${ }^{30} \mathrm{~b}$ ) los jardines y macetas con plantas, ${ }^{31} \mathrm{c}$ ) las imágenes de naturaleza, ${ }^{32} \mathrm{~d}$ ) la proximidad de áreas verdes exteriores. ${ }^{21}$ Asimismo, otras variables como e) la percepción de restauración ambiental y f) las actividades con la naturaleza ${ }^{33}$ han sido correlacionadas, en conjunto con las variables ambientales referidas, considerando indicadores fisiológicos, ${ }^{34}$ emocionales ${ }^{35} \mathrm{y}$ atencionales. ${ }^{36}$ Pese a que ya existen evidencias sobre el rol de estas variables en la promoción de la RP, poco se sabe de la evaluación de dichas variables en función de su posible influencia restauradora en un contexto de viviendas urbanas. Por tal motivo, en el presente trabajo se planteó como objetivo documentar el posible efecto restaurador de la naturaleza urbana residencial, ${ }^{37}$ considerando un modelo ecológico social del impacto de la naturaleza urbana en la restauración psicológica. En la fundamentación de dicho modelo se considera:

a) una orientación contextual y salutogénica que se relaciona con los procesos psicológicos y conductas que favorecen el bienestar físico y emocional, b) énfasis en las transacciones que ocurren entre el individuo y su entorno físico y social a través del tiempo, c) la naturaleza multifacética de las condiciones ambientales en la salud y bienestar de las 


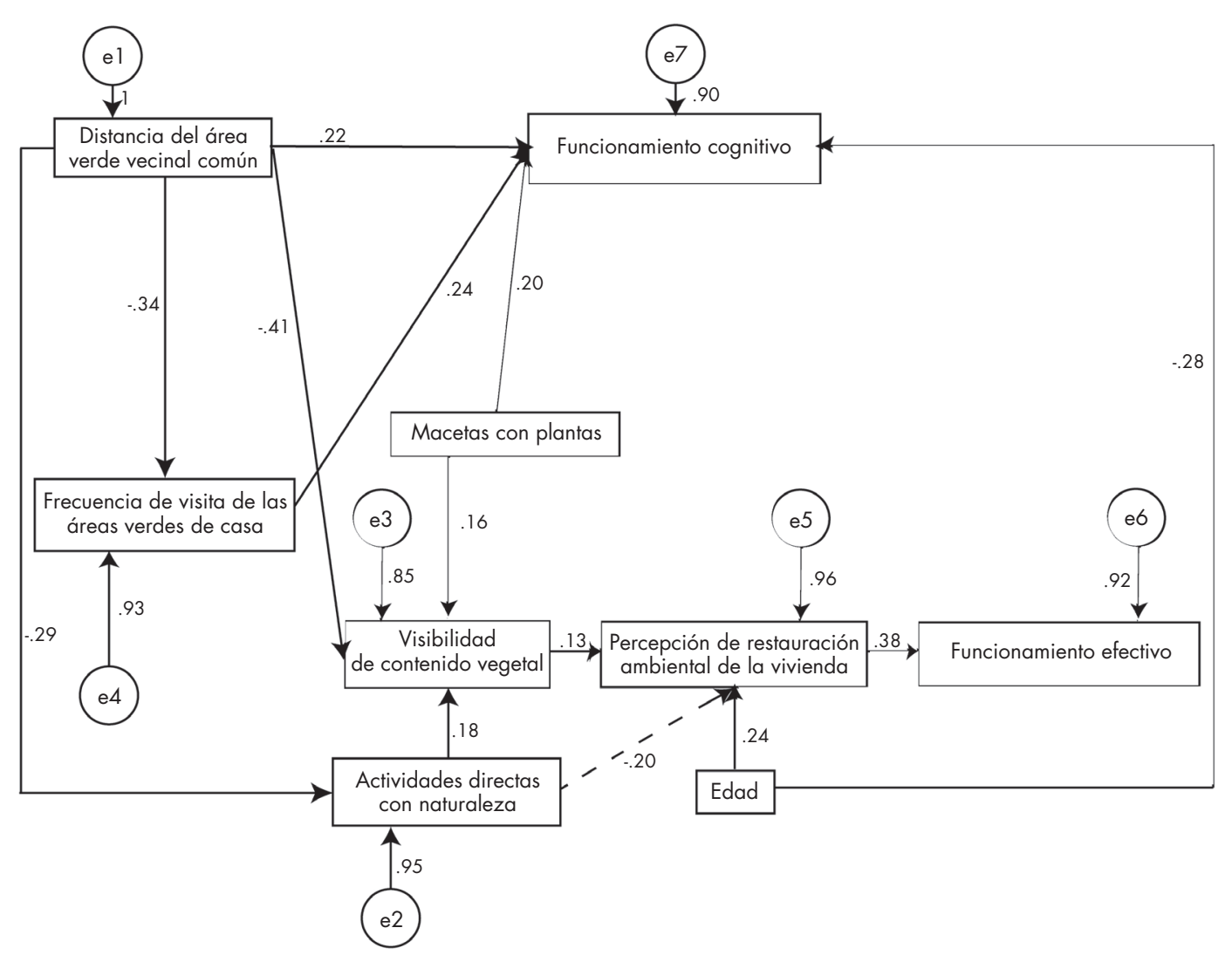

Figura 1. Modelo Ecológico Social del impacto de la Naturaleza Urbana en la restauración psicológica.

personas y d) la identificación de los recursos ambientales claves que probablemente influyan en el bienestar personal y colectivo entre los miembros de un escenario (exposición a ambientes restauradores-AR y sus efectos en las dimensiones de la RP). ${ }^{38}$ En la figura 1 se observa que la variable de NU comprende la dimensión de naturaleza en casa, la cual alude a la visibilidad de contenido vegetal desde las ventanas de la vivienda, los jardines y macetas con plantas e imágenes de naturaleza al interior de la vivienda, y proximidad de áreas verdes exteriores, que se refiere a la cercanía en tiempo y metros de la vivienda a las áreas verdes. PPTRAMA alude a las variables de percepción de restauración ambiental de la vivienda, que se refiere a la percepción de las cualidades restauradoras de la vivienda y las actividades con la naturaleza que comprenden conductas intencionales, circunstanciales, activas o pasivas, periódicas o esporádicas ${ }^{24}$ con la naturaleza. La RP representa dos dimensiones: el funcionamiento cognitivo, relacionado con la capacidad de concentración y atención dirigida y la dimensión emotiva, medida como incremento en el afecto positivo y una sensación de funcionamiento efectivo. ${ }^{39}$ A partir del modelo citado, que en adelante se le referirá como modelo Ecológico Social del impacto de la Naturaleza Urbana en la RP (EcSocNU), se derivó la siguiente pregunta de investigación: ¿Cuál es la magnitud y dirección de las relaciones existentes entre las variables de NU (naturaleza en casa y proximidad de áreas verdes exteriores) y PPTRAMA (percepción de restauración ambiental de la vivienda y actividades con la naturaleza), considerando las dos dimensiones de la RP (cognitiva y emotiva)?

En congruencia con la pregunta de investigación descrita, se derivaron tres hipótesis: a) Las variables de NU tendrán un impacto positivo y significativo en las dimensiones cognitivas y emotivas de la RP. b) Existirán relaciones positivas y significativas entre las variables de NU y PPTRAMA. c) Las variables de NU tendrán un impacto positivo y significativo en las dimensiones cognitivas y emotivas de la RP a través de los PPTRAMA.

\section{MÉTODO \\ Participantes}

Por medio de un muestreo intencional se efectuaron 120 entrevistas domiciliarias con residentes de una colonia de la parte oriente de la Zona Metropolitana del Valle de México (ZMVM). La edad de los participantes fluctuó entre 17 y 79 años, con edad promedio de 45.73 años ( $D E=14.23)$. Del total de la muestra, 63 (52.5\%) fueron hombres y $57(47.5 \%)$ mujeres. 


\section{Escenario de estudio}

La colonia encuestada, ubicada al oriente de la Ciudad de México, con código postal 57179, se eligió debido a que presenta algunas condiciones físicas y sociales con características homogéneas que permiten controlar posibles fuentes extrañas de variabilidad relacionadas con diferencias al ambiente físico y social. ${ }^{40}$ Entre los aspectos físicos se encuentran las cualidades de las viviendas: tamaño del terreno, el tipo de vivienda y la densidad habitacional. Respecto al tamaño del terreno, un $15.5 \%$ de las casas contaron con más de $80 \mathrm{~m}^{2}$ de terreno, mientras que el restante $84.5 \%$ se ubicaron en un rango de 60 a $80 \mathrm{~m}^{2}$ (moda $\left.=\mathrm{a} 75 \mathrm{~m}^{2}\right)$. En el tipo de vivienda, el $97.4 \%$ correspondió al diseño arquitectónico denominado "casa dúplex". En relación con los aspectos sociales, el nivel socioeconómico de la zona estuvo equilibrado considerando un nivel de clase media.*

\section{Instrumentos y mediciones}

Escala de Naturaleza en Casa (ENC). La ENC ${ }^{41}$ cuantifica en términos de porcentajes (nada=0 a 3\% hasta casi todo $97 \%$ a $100 \%$ ) la visibilidad de contenido vegetal observable a través de las ventanas de la vivienda y la visibilidad desde la calle. En una lista de chequeo se evaluó la presencia de macetas con plantas, jardines e imágenes de naturaleza (e.g posters) al interior de la casa. La validez de contenido se obtuvo en un estudio previo con la consulta de tres expertos en psicología ambiental y uno en ecología. En ese mismo estudio se evalúo la confiabilidad interjueces considerando la participación de tres expertos en psicología ambiental quienes evaluaron decenas de fotografías de ventanas con naturaleza y realizaron ocho sesiones extensivas de evaluación con la lista de chequeo en diferentes escenarios de vivienda. En dichas sesiones se obtuvo un porcentaje del $94 \%$ de acuerdos respecto a las cualidades a medir. Este criterio cumple con las expectativas de confiabilidad entre jueces que permiten emplear dicho instrumento en campo. ${ }^{42}$ Proximidad de áreas verdes exteriores. Se utilizó un podómetro digital con el que se registraba la distancia en tiempo y metros del área verde común más cercana a la casa de los participantes. Para fines de análisis se le describirá de aquí en adelante como distancia del área verde vecinal común. Escala de Transacciones con la Naturaleza (ETRAN). ${ }^{41}$ Se empleó para evaluar las actividades con la naturaleza. Es una escala unidimensional $(\alpha$ total $=0.61)$ con 11 ítems y un formato de respuesta de cinco opciones que van desde (0) nunca a (5) todos los días. El instrumento considera las actividades directas con la naturaleza ("practicar la jardinería") e indirectas ("escuchar discos compactos con sonidos de naturaleza"). Escala de Percepción de Restauración Ambiental Revisada, EPRA-R, ${ }^{43}$ se utilizó para medir la percepción de restauración ambien-

* SIGMA DOS MEXICO. Estudios de mercado. tal de la vivienda. Consta de 26 ítems escalares con opciones de respuesta $0=n a d a$ a 10 completamente y cinco factores: Fascinación $(\alpha=.81)$, Estar Alejado $(\alpha=.76)$, Compatibilidad $(\alpha=.75)$, Coherencia $(\alpha=.70)$ y Alcance $(\alpha=.81)$, así como dos ítems indicadores de preferencia ambiental. En cuanto a la validez de la EPRA-R, Martínez-Soto y Montero ${ }^{43}$ documentaron en un estudio previo evidencias de validez discriminante $^{44}$ y concurrente (mediante el empleo de una escala de estrés y activación). ${ }^{45}$ Subprueba de Retención de Dígitos en Orden Inverso. ${ }^{46}$ Se usó para evaluar la dimensión cognitiva de la RP en función de los cambios en la atención dirigida. ${ }^{47}$ La referida prueba depende de la atención dirigida debido a que los participantes deben moverse dentro y fuera de su foco atencional ${ }^{48}$ el cual es un componente mayor de la memoria a corto plazo. ${ }^{49}$ La prueba consiste en la lectura de una serie de siete pares de números aleatorios en orden creciente que son leídos al participante quien posteriormente debe repetirlos. El examinador al momento de evaluar las puntuaciones crudas debe considerar un rango de puntuación de 4 o 5 dentro de los límites normales, 3 como limítrofe o defectuoso, dependiendo de la educación del participante y 2 como defectuoso. ${ }^{50}$ Escala de Bienestar (EB) (adaptada de Kaplan ${ }^{23}$ por Martínez-Soto ${ }^{41}$ en población mexicana). Se empleó para evidenciar la dimensión emotiva de la RP. Consta de tres factores: Funcionamiento Efectivo $(\alpha=.84)$, Tranquilidad $(\alpha=.80)$ y Distracción $(\alpha=.72)$, con 26 ítems y cinco opciones de respuesta (0) nunca o rara vez a (4) muy frecuentemente.

\section{Procedimiento}

Los datos fueron recolectados por tres encuestadores (estudiantes de posgrado en psicología ambiental) que fueron exhaustivamente capacitados (tres horas en entrenamiento general de técnicas de recolección de datos, ocho horas en la recolección de los registros directos y 20 horas supervisadas en campo aplicando toda la batería de instrumentos). En la administración final, un entrevistador realizó los registros indirectos (variables psicológicas), mientras que un segundo encuestador o co-observador condujo una lista detallada de observaciones correspondientes a los registros directos relativos a la ENC y proximidad de áreas verdes exteriores. El tiempo promedio para levantar los registros fue de $45 \mathrm{mi}-$ nutos por vivienda.

\section{Análisis de datos}

Para ver en qué medida los datos se agruparon en torno a un valor central, se obtuvieron estadísticos descriptivos. Con la finalidad de conocer las relaciones directas e indirectas propuestas en el modelo EcSocNU se efectuó un análisis de sendas o trayectorias (Structural Equation Modeling, SEM) ${ }^{51}$ Para evaluar la bondad de ajuste se utilizó el índice de ajuste absoluto RMSEA $^{52}$ que requiere que el error no 
Cuadro 1. Estadísticas descriptivas para las variables de PPTRAMA y RP

\begin{tabular}{lccccc}
\hline Variable & $\begin{array}{c}\text { Dimen- } \\
\text { sión }\end{array}$ & $\mathrm{n}$ & Media & $\mathrm{DE}$ & $\begin{array}{c}\text { Rango de } \\
\text { respuesta }\end{array}$ \\
\hline Actividades con la & ADN & 119 & 1.51 & 0.65 & $0-5$ \\
Naturaleza & AIN & & 1.61 & 0.85 & $0-5$ \\
Percepción de res- & FA & & 7.32 & 1.74 & $0-10$ \\
tauración ambiental & EA & & 6.76 & 2.20 & $0-10$ \\
de la vivienda & COM & 119 & 8.47 & 1.57 & $0-10$ \\
& COH & & 7.84 & 1.82 & $0-10$ \\
& ALC & & 6.74 & 2.05 & $0-10$ \\
Restauración Psico- & FE & & 3.17 & 0.61 & $0-4$ \\
lógica indicadores & T & 119 & 2.53 & 0.79 & $0-4$ \\
emotivos & D & & 1.53 & 0.75 & $0-4$ \\
Restauración psico- & RDOI & & 4.22 & 1.27 & - \\
lógica indicador cog- & & 119 & & &
\end{tabular}

Nota: $A D N=$ Actividades directas con la naturaleza; $A I N=$ Actividades indirectas con la naturaleza, $\mathrm{FA}=$ Fascinación, $\mathrm{EA}=$ Estar alejado, $\mathrm{COM}=$ Compatibilidad; $\mathrm{COH}=$ Coherencia, $\mathrm{ALC}=$ Alcance, $\mathrm{FE}=$ Funcionamiento efectivo, $\mathrm{T}=$ Tranquilidad, $\mathrm{D}=$ Distracción; $\mathrm{RDOl}=$ Retención de dígitos en orden inverso.

rebase .05. Como índices de incremento o nivel de ajuste se consideraron tres: (a) CFI (Comparative Fit Index), (b) TLI (Tucker-Lewis Index) y (c) NFI (Normed Fit Index). El criterio de aceptación para estos índices fue de .90 a 1.00.53

\section{RESULTADOS}

En el cuadro 1 se presentan las estadísticas descriptivas de las variables.

De los 120 domicilios evaluados mediante la ENC se encontró que poco menos de la mitad $(42.9 \%)$ contaba con algún jardín en el hogar. Asimismo, la visibilidad de naturaleza desde la calle fue una de las perspectivas en donde se podía ver mayor parte de ésta, seguido de las vistas de la sala y la recámara. Se encontraron promedios escalares bajos (escala de 0 a 2 ) tanto para las imágenes de naturaleza $(M=.45 ; D E=.37)$ como para las macetas con plantas $(M=1.32 ; D E=.65)$. En cuanto a la distancia del área verde vecinal u otra área verde menor, se encontró que en promedio dichas áreas están a menos de tres minutos de las casas encuestadas. Por otra parte, la mayoría de las personas reportó visitar con relativa frecuencia las áreas verdes cercanas a su vivienda (42.5\%) mientras que un $14.5 \%$ refirió que nunca las visitaba. Respecto a las actividades con la naturaleza se encontraron puntuaciones debajo del promedio escalar (escala de 0 a 5, $M=1.56, D E=.75$; cuadro 1). Las medias escalares para las cualidades restauradoras de la vivienda por lo general estuvieron por encima del promedio $(M=7.42, D E=.73)$ escala de 0 a 10 . En relación a las dimensiones emotivas de la RP, el factor de Funcionamiento Efectivo de la EB obtuvo la media escalar más alta, seguido

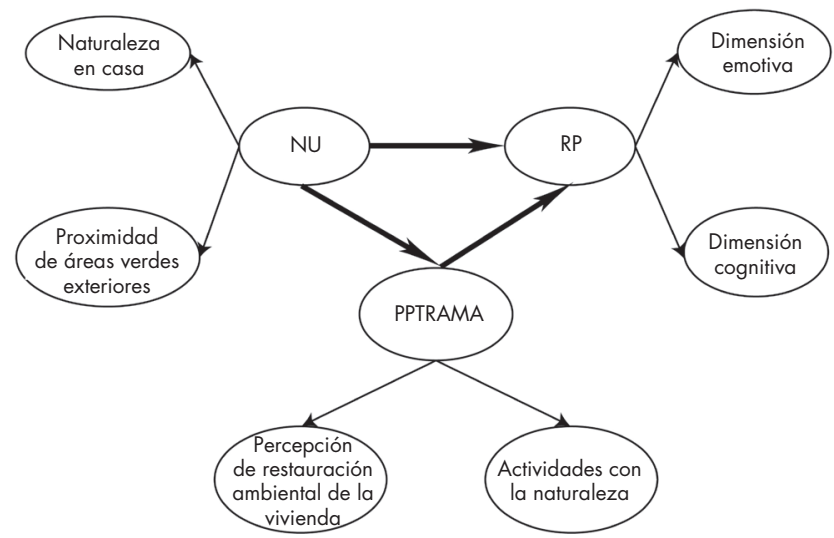

Figura 2. Modelo de la influencia restauradora de la naturaleza urbana. Indicadores de bondad de ajuste $X^{2}=29.56, g l=23, p=.26$, $\mathrm{NFI}=.78$ y $\mathrm{RMSEA}=.05$.

de Tranquilidad y por último Distracción. Por otra parte, en el aspecto de funcionamiento cognitivo representado por la Subprueba de Retención de Dígitos en Orden Inverso, se obtuvo una media de 4.22, puntuación que se aproxima a los promedios normales para una población de adultos. ${ }^{50}$

\section{Análisis de trayectorias}

En el modelo empírico se incluyeron variables de al menos una de cada una de las categorías generales del modelo teórico EcSocNU (figura 1), con lo que fue posible evidenciar un ajuste teórico y empírico proveniente de los datos. El modelo quedó integrado por nueve de 16 variables observadas (figura 2) considerando una muestra de 113 casos que cumplieron con el criterio de no contar con algún valor perdido. Dicho tamaño muestral cumple con los criterios mínimos de parámetros y casos a considerar en un modelamiento estructural. ${ }^{54}$

Los índices obtenidos para el modelo EcSocNU fueron de $X^{2}=29.56, g l=23, p=.16$, del cual se desprende que el modelo tiene bondad de ajuste a los datos, ya que, en este caso, el valor de $X^{2}$ es pequeño y la probabilidad asociada no es significativa. ${ }^{55} \mathrm{El}$ índice de ajuste absoluto RMSEA fue de .05, mismo que se encuentra dentro de los límites que corresponden a un buen ajuste. ${ }^{56}$ En cuanto a los índices de ajuste de incremento se reportaron valores de $\mathrm{CFI}=.93$; $\mathrm{TLI}=.90$ y NFI=.78. Este último valor, a diferencia de los demás indicadores que son más estables, no obtuvo los valores deseables (>.90), sin embargo, el hecho de considerarlo como un indicador que es sensible al tamaño de la muestra permite asumir con cautela que el modelo tiene un buen ajuste. ${ }^{51}$ Por otra parte, el valor de $\mathrm{R}^{2}$ obtenido fue de .15 para funcionamiento efectivo y de .19 para funcionamiento cognitivo. Los valores correspondientes a los errores estandarizados mostraron valores desde 1 hasta el valor de .85 . Estos resultados indican la importancia de los hallazgos en las relaciones de las variables estudiadas con las precaucio- 
nes de interpretación que imponen los valores de error de medición. ${ }^{52}$

Las variables observadas que predijeron positiva y significativamente el funcionamiento cognitivo fueron: macetas con plantas (.20) y la frecuencia de visita de las áreas verdes fuera de casa (.24). Por otra parte, de manera inesperada se encontró que a mayor distancia del área verde vecinal común (.22) hubo un mejor funcionamiento cognitivo.

Como se observa en la figura 2, varios senderos inician con la distancia del área verde vecinal común. Dicha variable también tuvo un impacto indirecto sobre el funcionamiento cognitivo a través de la frecuencia de visita de las áreas verdes fuera de casa (-.34). La variable de percepción de restauración ambiental de la vivienda (se consideró la puntuación promedio global de los cinco factores de la EPRA-R) fungió como variable mediadora en la relación $\mathrm{NU}$ y efecto restaurador del tipo emotivo. La visibilidad de contenido vegetal presentó una correlación inversa con la distancia del área verde vecinal común (-.41), y directa con la presencia de macetas con plantas (.16) para posteriormente incidir en la percepción de restauración ambiental de la vivienda (.13) y finalizar en el funcionamiento efectivo (.38).

Otro de los senderos encontrados correspondió a la correlación inversa de la distancia del área verde vecinal común con las actividades directas con la naturaleza (-.29), sin embargo ésta no tuvo un efecto sobre la percepción de restauración ambiental de la vivienda. Finalmente cabe señalar la influencia positiva que tuvo la edad sobre la percepción de restauración ambiental de la vivienda (.24) y la relación negativa de ésta sobre el funcionamiento cognitivo (-.28), lo que sugiere que a mayor edad, hay un peor funcionamiento cognitivo. ${ }^{57}$

\section{DISCUSIÓN}

En el presente estudio se partió de la necesidad de evidenciar que existen factores del ambiente físico que pueden repercutir positivamente en la salud mental. A partir de una aproximación ecológica social ${ }^{38}$ y derivado del estudio de los AR y de la $\mathrm{RP}^{24,28}$ se propuso un modelo Ecológico Social del impacto de la Naturaleza Urbana en la RP (EcSocNU). Por medio del referido modelo se documentaron las relaciones entre variables de NU y PPTRAMA considerando su impacto en dos dimensiones de la RP. En una primera hipótesis se planteó que las variables de NU tendrían un impacto positivo y significativo en las dimensiones de la RP. Al respecto se demostró la existencia de los efectos positivos y significativos de la variable de macetas con plantas sobre la dimensión cognitiva de la RP, lo cual también coincide con investigaciones anteriores. ${ }^{23,58}$ Asimismo, se ratificó que la frecuencia de visitas a las áreas verdes exteriores de casa tuvo un impacto positivo sobre el funcionamiento cognitivo (.24). De manera imprevista, se encontró que a mayor distancia del área verde vecinal común hay un mejor funcionamiento cognitivo. Esta relación negativa nos recuerda algunos de los principios relevantes a considerar en el estudio de los $\mathrm{AR}^{41}$ que tienen que ver con la temporalidad en la exposición a los AR. El mismo tipo de ambiente puede no ser restaurador para todas las personas y no serlo todo el tiempo; más aún, el tiempo de exposición considerado de manera aislada no garantiza la restauración psicológi$\mathrm{ca}^{59}$ por lo que en un impacto positivo de la distancia de las áreas verdes y el funcionamiento cognitivo valdría la pena considerar la influencia de otras variables psicológicas, por ejemplo, la necesidad de restauración individual ${ }^{60} \mathrm{y}$ las actitudes hacia las áreas verdes urbanas. ${ }^{61}$ En ciertos casos las áreas verdes vecinales pudieran ser percibidas como amenaza para algunos residentes debido a que oculten fauna nociva o sean percibidos como escenarios para la delincuencia y sitios de contaminación por desechos sólidos. Asimismo, independientemente del contacto físico directo con la naturaleza, evidencias en el campo de las neurociencias sugieren que, en la conformación de las experiencias restaurativas, la memoria episódica y la imaginería visual pueden desempeñar, al igual que la percepción visual, un rol activo en la conformación de la RP.* En una segunda hipótesis se planteó la existencia de relaciones positivas y significativas entre las variables de NU y PPTRAMA. En este sentido se documentó a la visibilidad de contenido vegetal como una variable crítica para la promoción de la percepción de restauración ambiental de la vivienda, lo cual es congruente con investigaciones previas. ${ }^{26,29}$ En una tercera hipótesis se propuso que las variables de NU tendrían un impacto positivo y significativo en las dimensiones de la RP, a través de las variables de PPTRAMA. Se documentó la percepción de la restauración ambiental de la vivienda como un aspecto que media la relación entre la NU (ej. distancia del área verde vecinal focal, visibilidad y macetas con plantas) y la $\mathrm{RP}$ en su indicador emotivo de funcionamiento efectivo. Las teorías sobre restauración psicológica ambiental ${ }^{62,63}$ no suelen ser muy claras respecto al rol que pueden ejercer algunas variables sociodemográficas (e.g. género y edad) en la percepción de las cualidades restauradoras del ambiente. Dentro del modelo empírico EcSocNU se aprecia la relación entre edad y percepción de restauración ambiental de la vivienda. Algunos estudios sugieren que los cambios en los ciclos de vida de las personas pueden impactar la percepción que se tenga sobre los componentes restaurativos. ${ }^{64} \mathrm{En}$ esta investigación se encontró que a mayor edad hay una mayor percepción de restauración ambiental de la vivienda; de lo que se desprende que los componentes restauradores son valorados con mayor intensidad por los adultos al compararse con los jóvenes. ${ }^{43}$ Como la mayoría de los estudios * Martínez-Soto, J. Gonzáles-Santos, L. \& Barrios, F. 2012. Psychological
Restoration: an evaluation of some neural correlates. 22 IAPS Conference, Glasgow, Escocia, UK. 
dentro de esta área de estudio poco explorada de RP en escenarios urbanos considerando un contexto de vivienda, en el presente trabajo se identifican algunas limitaciones que abren nuevas líneas de investigación. Los resultados obtenidos aluden a las experiencias restauradoras de la gente dentro de sus propios escenarios y no en condiciones de laboratorio. Si bien es deseable el control de las variables como medio para incrementar la certeza de las inferencias generadas, no siempre es factible reproducir en el laboratorio las condiciones prevalecientes en un escenario natural, tal es el caso de la restauración ambiental. En la presente investigación no se incluyeron medidas fisiológicas que evidenciaran la RP. Investigaciones futuras podrían considerar este tipo de registros. El valor obtenido en términos del porcentaje de varianza explicada para el modelo fue de $14 \%$. El poco poder predictivo alcanzado en el modelo ejemplifica la complejidad que implica la evaluación de la influencia restauradora de la naturaleza urbana. Se sugiere que en futuros estudios se considere la inclusión de otras variables que podrían maximizar la varianza sistemática, entre ellas la necesidad de restauración de las personas, las actitudes hacia las áreas verdes, el tiempo de exposición real con la naturaleza (dosis de naturaleza-beneficios psicológicos obtenidos) y posibles factores de habituación a las áreas verdes. ${ }^{41}$ Los errores estandarizados altos dentro del modelo, mismos que implican otras influencias no especificadas, ${ }^{65}$ podrían minimizarse con la eliminación de fuentes de cansancio (empleo de baterías de instrumentos más cortas) y la incorporación de más participantes en la muestra. ${ }^{56}$ Asimismo, este tipo de variabilidad puede reducirse al incorporar otro conjunto de variables no contempladas inicialmente en el modelo. ${ }^{56}$

Por otra parte, en concordancia con una aproximación ecológica social se utilizaron metodologías diversas (e.g. registros directos e indirectos) para evaluar los escenarios intra y extra-vivienda, ${ }^{66}$ lo que constituye una aportación metodológica novedosa para el estudio de la RP en escenarios urbanos residenciales. El estudio de los AR y de la RP constituyen líneas de investigación relevantes para la promoción de la salud mental. Los efectos restaurativos aluden a una reducción en los niveles de estrés, promoción de una mejor capacidad de concentración y atención dirigida, estados positivos del humor y una sensación de funcionamiento efectivo. ${ }^{20,21} \mathrm{La}$ salud mental contempla el goce de un equilibrio emocional y cognitivo, el bienestar subjetivo, una sensación de funcionamiento efectivo y una capacidad de encarar adecuadamente el estrés. La influencia restauradora de la naturaleza urbana puede constituir uno de los beneficios psicológicos más accesibles para la promoción de la salud mental en habitantes urbanos. En este sentido, futuros estudios podrían documentar la relación entre los efectos restaurativos del contacto con la naturaleza y evaluaciones de salud mental. ${ }^{11}$ Asimismo, en el ámbito de la salud pública es importante valorar la conservación de naturaleza en camellones y otras vialidades de importancia, pues según lo encontrado, son aspectos que promueven beneficios psicológicos importantes. En síntesis, de los hallazgos obtenidos se derivan las siguientes implicaciones: El contacto con elementos naturales puede promover la salud mental; la generación de escenarios urbanos debería ser equilibrado con el acceso a espacios naturales; la psicología puede colaborar conjuntamente con disciplinas como la medicina, la ecología, el urbanismo, entre otras, en el desarrollo de instrumentos que documenten el impacto que las cualidades físicas del ambiente - ej. naturaleza urbana-, ejercen sobre la salud física y mental de los individuos. La aproximación ecológica-social ofrece una plataforma conceptual a partir de la cual desarrollar investigación innovadora con implicaciones empíricas relevantes para la promoción de la salud mental. De estas implicaciones se desprende que la psicología puede contribuir al desarrollo de políticas públicas de salud que promuevan el óptimo funcionamiento tanto del individuo como de las comunidades.

\section{AGRADECIMIENTOS}

El estudio del que se derivó el presente artículo fue parcialmente apoyado por el CONACyT con una beca (No. 201642) para estudios doctorales de Joel Martínez-Soto, bajo la supervisión de la doctora María Montero y López-Lena. Se agradece el apoyo técnico del maestro Leopoldo Gonzáles-Santos, del Instituto de Neurobiología, Juriquilla, UNAM.

\section{REFERENCIAS}

1. Kaminoff R, Proshansky H. Stress as a consequence of the urban physical environment. En: Goldenber L, Breznitz S (eds). Handbook of stress: Theoretical and clinical aspects. New York: MacMillan Publishing; 1982.

2. Krabbedan L, van Os J. Schizophrenia and urbanicity: a major environmental influence-conditional on genetic risk. Schizophr Bull 2005;31:795-799.

3. OPS. Cartografía de la salud mental: datos básicos. Consultado en: http://www.paho.org/Spanish/DD/PIN/saludmental_005.htm el 11 de febrero 2012.

4. Medina-Mora ME, Borges G, Lara C, Benjet C et al. Prevalencia de trastornos mentales y uso de servicios: Resultados de la Encuesta Nacional de Epidemiología Psiquiátrica en México. Salud Mental 2003;26(4):1-16.

5. Booth-Kewley S, Friedman H. Psychological predictors of heart disease: A quantitative review. Psychol Bull 1987;101(3):343-362.

6. Watson D, Pennebaker J. Health complaints, stress, and distress: Exploring the central role of negative affectivity. Psych Rev 1989;96:234-254.

7. O'Donnell M, Harris J. Health promotion in the workplace. Nueva York: Delmar Publishers; 1994.

8. Collazos M. Trastornos mentales y problemas de salud mental. Día Mundial de la Salud Mental 2007. Salud Mental 2007;30(2):75-80.

9. Wagner F, Gonzales-Forteza C, Sánchez-García S, García-Peña C et al. Enfocando la depresión como problema de salud pública en México. Salud Mental 2012;35:3-11.

10. Organización Mundial de la Salud. El informe mundial de la salud 2001. Nuevos conocimientos, nuevas esperanzas. Suiza, Ginebra: 2001.

11. Lluch M. Evaluación empírica de un modelo conceptual de salud mental positiva. Salud Mental 2002;25(4):42-55.

12. De la Fuente JR. ¿Hacía donde va la investigación en psiquiatría? Salud Mental 1995;18(2):55-59.

13. OMS. Temas de salud mental. Consultado en: http://www.who.int/topics/mental_health/es/index.html el 11 febrero 2012.

14. Organización Mundial de la Salud. Promoción de la salud mental. Conceptos, evidencia emergente y práctica. Génova: 2004. 
15. Secretaria de Salud. Programa de acción específico 2007-2012. Atención en salud mental. México: 2008.

16. Frumkin H. Beyond toxicity: human health and the natural environment. American J Preventive Medicine 2001;20:234-240.

17. Jahoda M. Current concepts of positive mental health. Nueva York: Basic Books; 1958.

18. Werner C, Brown B, Altman I. Transactionally oriented research: Examples and strategies. En: Bechtel R, Churchman A (eds). Handbook of environmental psychology. New York, NY: John Wiley \& Sons; 2002.

19. Martínez-Soto J, Gonzáles-Santos L, Barrios F. Aplicación de una versión computarizada para evaluar las cualidades afectivas del ambiente. Aportaciones Actuales Psicología Social 2012;1:476-480.

20. Park JB, Furuya $K$, Kasetani $T$, Takayama $\mathrm{N}$ et al. Relationship between psychological responses and physical environments in forest settings. Landscape Urban Planning 2011;102:24-32.

21. Ryan R, Weinstein N, Bernstein J, Brown $K$ et al. Vitalizing effects of being outdoors and in nature. J Environmental Psychology 2010;30:159-168.

22. Herzog TR, Strevey SJ. Contact with nature, sense of humor, and psychological well-being. Environment Behavior 2008;40(6):747-776.

23. Kaplan R. The nature of the view from home: Psychological benefits. Environment Behavior 2001;33:507-542.

24. Kaplan S, Talbot J. Psychological benefits of a wilderness experience. En: Altman I, Wohlwill JF (eds). Behavior and the natural environment. New York: Plenum; 1983.

25. Payne S. Are perceived soundscapes within urban parks restorative? J Acoustical Society America 2008;123(5):3809.

26. Ulrich R. View through a window may influence recovery from surgery. Science 1984;224:420-421.

27. Berto R. Exposure to restorative environments helps restore attentional capacity. J Environmental Psychology 2005;25:249-259.

28. van den Berg A, Hartig T, Staats H. Preference for nature in urbanized societies: Stress, restoration, and the pursuit of sustainability. J Social Issues 2007;63:79-96.

29. Hernández B, Hidalgo C. Effect of urban vegetation on psychological restorativeness. Psychol Rep 2005;96:1025-1028.

30. Raanaas R, Evensen K, Rich D, Sjøstrøm, G, Patil, G. Benefits of indoor plants on attention capacity in an office setting. J Environmental Psychology 2011;31:99-105.

31. Bringslimark T, Hartig T, Patil G. The psychological benefits of indoor plants: A critical review of the experimental literature. J Environmental Psychology 2009;29:422-433.

32. Parsons R, Tassinary L, Ulrich R, Hebl M et al. The view from the road: Implications for stress recovery and immunization. J Environmental Psychology 1998;18:113-140.

33. Cimprich B. Development of an intervention to restore attention in cancer patients. Cancer Nurs 1993;16:83-92.

34. Hartig T, Evans GW, Jamner LD, Davis D et al. Tracking restoration in natural and urban field settings. J Environmental Psychology 2003;23:109-123.

35. Kweon B, Ulrich R, Walker V, Tassinary L. Anger and stress. The role of landscape posters in office settings. Environment Behavior 2008;40:355-381.

36. Velarde MD, Fry G, Tveit M. Health effects of viewing landscapesLandscape types in environmental psychology. Urban Forestry Urban Greening 2007;6:199-212.

37. Ballester OJ, Morata CA. Normas para la clasificación de los espacios verdes. España: Universidad Politécnica de Valencia; 2001.

38. Montero LLM, Evans G. Perspectiva ecológica social, una opción heurística para el estudio de la pobreza. En: Montero M, Mayer D (eds). Ecología social de la pobreza: impactos psicosociales, desafíos multidisciplinarios. México: UNAM; 2010.

39. Valtchanov D, Barton K, Ellard C. Restorative effects of virtual nature settings. Cyberpsychology Behavior Social Networking 2010;13(5):503-512.

40. Kuo F. Coping with poverty. Impacts of environment and attention in the inner city. Environment Behavior 2001;33:5-34.
41. Martínez-Soto J. Impacto de la naturaleza urbana próxima: un modelo ecológico social. Tesis de doctorado. México: Facultad de Psicología: UNAM; 2010.

42. Bechtel R, Zeisel J. Observation: The world under a glass. En: Bechtel RB, Marans RW, Michelson W (eds). Methods in environmental and behavioral research. New York: Van Nostrand Reinhold Co.; 1987.

43. Martínez-Soto J, Montero LLM. Escala de restauración ambiental percibida- EPRA: diferencias por edad y sexo en muestras de adolescentes y adultos mexicanos. En: Rodríguez B, Chapin M (eds). Linking differences/defining actions. Oklahoma: EDRA; 2008.

44. Hartig T, Korpela K, Evans GW, Gärling T. A measure of restorative quality in environments. Scandinavian Housing Planning Research 1997;14:175-194.

45. King M, Burrows G, Stanley G. Measurement of stress and arousal: Validation of the stress/arousal adjective checklist. Br J Psychol 1983;74:473-479.

46. Wechsler D. Wechsler adult intelligent scale (WAIS-III). México: El Manual Moderno; 2004.

47. Berman M, Jonides J, Kaplan S. The cognitive benefits of interacting with nature. Psychological Science 2008;19(12):1207-1212.

48. Cowan N. The magical number 4 in short memory: A reconsideration of mental storage capacity. Behav Brain Sci 2001;24:87-114.

49. Jonides J. Voluntary vs. automatic control over the mind's eye's movement. En: Long JB, Badeley AD (eds). Attention and performance. Hillsdale, NJ: Erlbaum; 1981.

50. Baron IS. Neuropsychological evaluation of the child. USA: Oxford University Press; 2004.

51. Bentler PM. EQS 6 structural equations program manual. Encino, CA.: Multivariate Software, Inc.; 2006.

52. Klem L. Structural equation modeling. En: Grimm L, Yarnold P (eds). Reading and understanding multivariate statistics. Washington: American Psychological Association; 2000.

53. MacDonald PR, Ho RM. Principles and practice in reporting structural equation analyses. Psychological Methods 2002;7:64-82.

54. Kline R. Principles and practice of structural equation modeling. New York: The Guilford Press; 2005.

55. Hoyle R. Structural equation modeling. Concepts, issues, and applications. Thousands Oaks, California: SAGE Publications; 1965.

56. Corral V. Aplicaciones del modelamiento estructural a la investigación psicológica. Revista Mexicana Psicología 2001;18:193-209.

57. Roselló J, Munar E. El mecanismo atencional: estudio de las diferencias individuales. Revista Psicología General Aplicada 1994;47:383-390.

58. Wells N. At home with nature -Effects of greenness on children's cognitive functioning. Environment Behavior 2000;32:775-795.

59. Hartig T, Staats H. Guest editors' introduction: Restorative environments. J Environmental Psychology 2003:23;103-107.

60. Smolders $K$, de Kort $Y$, Tenner A, Kaiser F. Need for recovery in offices: Behavior-based assessment. J Environmental Psychology 2012;32:126-134.

61. Ozguner H, Kendle D. Public attitudes toward naturalistic versus designed landscapes in the city of Sheffield (UK). Landscape Urban Planning 2006;74:139-157.

62. Kaplan S. The restorative benefits of nature: Toward an integrative framework. J Environmental Psychology 1995;15:169-182.

63. Ulrich R. Aesthetic and affective response to natural environment. En: Altman I, Wohlwill JF (eds). Human behavior and environment: Advances in theory and research. New York: Plenum Press; 1983.

64. Scopellitu M, Giuliani M. Choosing restorative environments across the lifespan: A matter of place experience. J Environmental Psychology 2004;24:423-437.

65. Loehlin J. Latent variables models. An introduction to factor, path and structural equation analysis. Hillsdale, NJ: Lawrence Erlbaum Associates; 2004.

66. Winkel G, Saegert S, Evans GW. An ecological perspective on theory, methods, and analysis in environmental psychology: Advances and challenges. J Environmental Psychology 2009;29:318-328. 